\title{
Propagation loss in photonic crystal waveguides embedding InAs/GaAs quantum dots determined by direct spectral imaging
}

Lorenzo Scarpelli, Ben Lang, Francesco Masia, Daryll Beggs, Egor Muljarov, et al.

Lorenzo Scarpelli, Ben Lang, Francesco Masia, Daryll Beggs, Egor Muljarov, Andrew B. Young, Ruth Oulton, Sven Höfling, Christian Schneider, Wolfgang Langbein, "Propagation loss in photonic crystal waveguides embedding InAs/GaAs quantum dots determined by direct spectral imaging," Proc. SPIE 10916, Ultrafast Phenomena and Nanophotonics XXIII, 1091617 (27 February 2019); doi: 10.1117/12.2510478

SPIE. Event: SPIE OPTO, 2019, San Francisco, California, United States 


\title{
Propagation loss in photonic crystal waveguides embedding InAs/GaAs quantum dots determined by direct spectral imaging
}

\author{
Lorenzo Scarpelli ${ }^{\mathrm{a}}$, Ben Lang ${ }^{\mathrm{b}}$, Francesco Masia ${ }^{\mathrm{a}}$, Daryl Beggs ${ }^{\mathrm{a}}$, Egor Muljarov ${ }^{\mathrm{a}}$, Andrew B. \\ Young $^{\mathrm{b}}$, Ruth Oulton ${ }^{\mathrm{b}}$, Sven Höfling ${ }^{\mathrm{c}}$, Christian Schneider ${ }^{\mathrm{c}}$, and Wolfgang Langbein ${ }^{\mathrm{a}}$ \\ aSchool of Physics and Astronomy, Cardiff University, The Parade, CF24 3AA, Cardiff, United \\ Kingdom \\ ${ }^{\mathrm{b}}$ Department of Electrical and Electronic Engineering, University of Bristol, Merchant \\ Venturers Building, Woodland Road, BS8 1UB, Bristol, United Kingdom \\ ${ }^{c}$ Technische Physik, Physikalisches Institut and Wilhelm Conrad Röntgen-Center for Complex \\ Material Systems, Universität Würzburg, Am Hubland, 97474, Würzburg, Germany
}

\begin{abstract}
We determine the propagation loss of GaAs photonic crystal waveguides by spectral imaging of the spontaneous emission from the embedded InAs/GaAs quantum dots. The results are compared with the loss obtained by imaging the near field of the out-of-plane radiation of the waveguide mode propagating within the light cone. From the corresponding far field, we furthermore measure the mode wavevector, from which we determine the waveguide dispersion. Additionally, we show that spectral imaging allows to determine the relative efficiencies of the couplers. Using the same experiment, and detailed photonic simulations, we have determined the beta factor and the directionality of the emission of the QDs, finding beta factors up to $99 \%$ and high directionalities.
\end{abstract}

Keywords: Photonic crystal waveguide, propagation loss, quantum dots, spectral imaging

\section{INTRODUCTION}

Photonic nanostructures allow the engineering of light propagation at the nanoscale, and are therefore a promising technology for building photonic networks. Furthermore, fabrication methods are compatible with the growth of self-assembled quantum dots (QDs) ${ }^{1}$ and site-controlled pyramidal QDs, ${ }^{2}$ which allowed quantumelectrodynamics investigations using these single photon emitters. In particular, photonic crystal waveguides (WG) embedding QDs have attracted attention, due to the broadband coupling ${ }^{3}$ and high beta factors ${ }^{4}$ which are a prerequisite for all-on-chip single photon sources. Additionally, directional emission of photons ${ }^{5}$ has been recently demonstrated, ${ }^{6,7}$ and exploited for spin-controlled photon switching ${ }^{8}$ an important step forward in the field of optical quantum information processing. All the above mentioned applications require low propagation loss. Although a WG mode confined within the photonic bandgap propagates with no loss, scattering of light due to fabrication imperfections couple the propagating mode to radiative modes within the light cone, resulting in propagation loss.$^{9,10}$ Propagation loss in photonic crystal WG have been determined by transmission measurements ${ }^{11,12}$ although from these measurements it is difficult to clearly identify the WG mode. An alternative method used high reflectors at both ends of the WG to form a resonator, with a finesse related to the propagation loss coefficient $\alpha .{ }^{13,14}$ Here we determine the loss from spectral imaging. The spontaneous emission (SE) of QDs is imaged onto the input slit of an imaging spectrometer, and is measured both spatially resolved along the WG and spectrally resolved. By scanning the excitation position along the WG, we excite QDs with different propagation distances to the bottom and top grating out-couplers, so that $\alpha$ can be determined from the ratio of the corresponding spectrally integrated intensities. Additionally, the ratio is proportional to the relative efficiency of the couplers, which can also be determined. We verify our method by comparing the loss coefficient obtained from spectral imaging, with the one measured by imaging the near field (NF) along the WG for an injected laser

Send correspondence to Wolfgang Langbein, E-mail: LangbeinWW@Cardiff.ac.uk, Telephone: +44 (0) 2920870172

Ultrafast Phenomena and Nanophotonics XXIII, edited by Markus Betz,

Abdulhakem Y. Elezzabi, Proc. of SPIE Vol. 10916, 1091617 • (C) 2019 SPIE

CCC code: $0277-786 \mathrm{X} / 19 / \$ 18 \cdot$ doi: $10.1117 / 12.2510478$

Proc. of SPIE Vol. 10916 1091617-1 
(a)

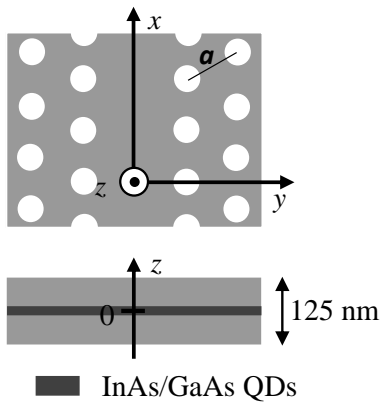

(b)

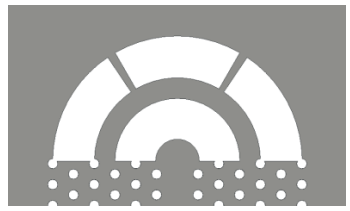

(c)

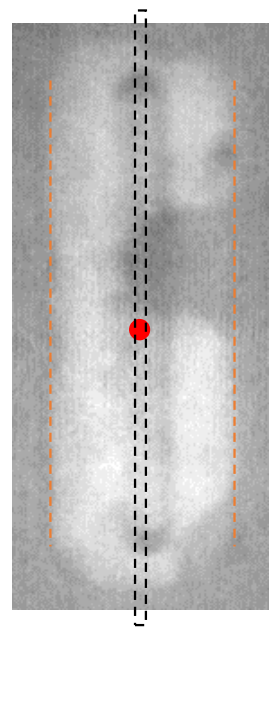

(d)

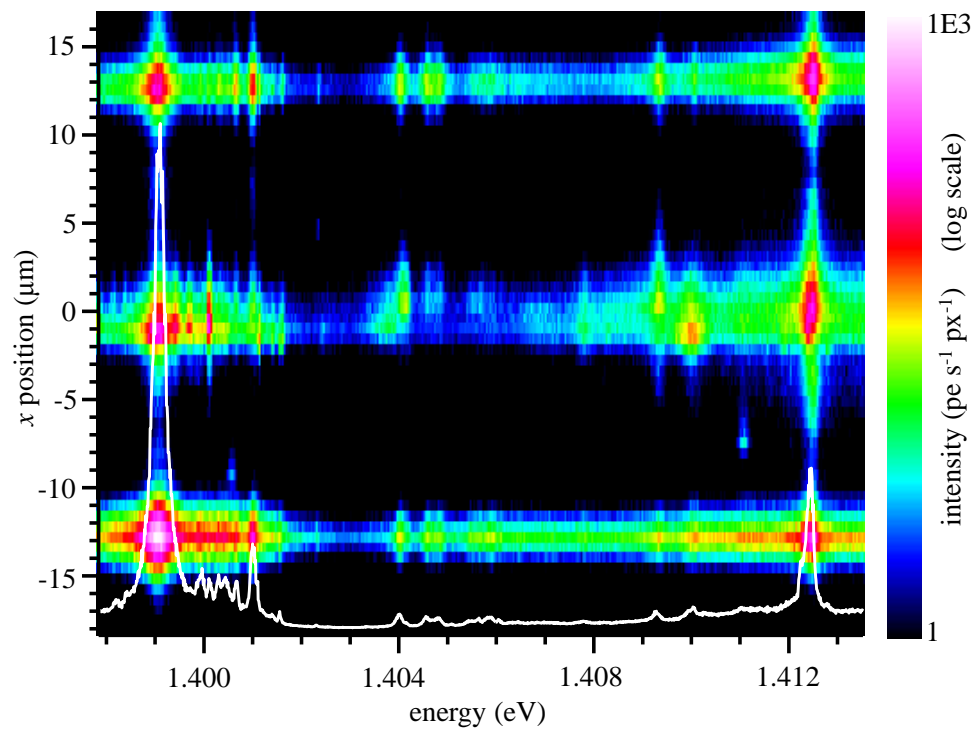

Figure 1. Waveguide structure and imaging. (a) Sketch of in-plane structure (top), and side view (bottom) of the GaAs membrane WG with embedded QDs. (b) Sketch of the grating coupler. (c) White light reflection image of the investigated WG; the spectrometer input slit region is indicated by the black dashed line; dashed orange lines: lateral edges of the underetched region, providing a free-standing GaAs membrane; red dot: excitation position. (d) Spectral image from the CCD camera range, in units of photoelectrons (pe) per second per pixel. The bottom and top spectra are the QDs SE into the WG mode and coupled into free space by the bottom and top grating out-couplers. The central spectrum is the QD free space emission; white line: spectrum emitted from the bottom grating coupler.

pulse, and we show agreement in the region of spectral overlap. By measuring the corresponding far field (FF), we determine the WG mode wavevector as a function of energy, and thus the WG dispersion inside the light cone. We note that using the same experiment, and detailed photonic simulations, we have determined the beta factor and the directionality of the emission of the QDs ${ }^{15}$ finding $99 \%$ beta factors and high directionalities. Furthermore, using beta factor and directionality, we estimated the position of the QD within the WG unit cell.

\section{SAMPLE AND EXPERIMENT}

The investigated sample features an hexagonal pattern of air holes of radius $r$ in a $125 \mathrm{~nm}$ thick GaAs membrane, with a lattice constant $a=260 \mathrm{~nm}$ and $r / a=0.26$. A line of holes is removed to form a $26 \mu \mathrm{m}$ long WG, terminated by Bragg output couplers. Sketches of few unit cells of the structure, and the coupler, are shown in Fig. 1a and Fig. 1b, respectively. At $z=0$, a layer of InAs is deposited in order to form InAs/GaAs QDs on a wetting layer (WL) by Stranski-Krastanov type self assembly, with a density of roughly $10^{9} \mathrm{~cm}^{-2}$. Deltadoping of n-type is provided by silicon atoms deposited $10 \mathrm{~nm}$ underneath the QD layer, with a doping density of $8 \times 10^{9} \mathrm{~cm}^{-2}$. A white light reflection image of the investigated WG is shown in Fig. 1c. The GaAs membrane side edges are highlighted by the orange dashed lines. The line defect is at $y=0$ and it has six lines of holes on each side, for a total lateral size of about $2.7 \mu \mathrm{m}$. The WG is terminated by grating couplers, as shown in Fig. 1b, which couple WG modes with free space modes of excitation and emission. The sample is mounted in a low vibration closed cycle cryostat (Montana Cryostation C2) on a XYZ piezo stage (Attocube) with a spatial resolution around $0.1 \mu \mathrm{m}$, allowing focusing and lateral alignment. All measurements are performed with a magnetic field $B=0.45 \mathrm{~T}$ in Faraday configuration, and at the sample temperature of $5 \mathrm{~K}$. A microscope objective (MO) with a numerical aperture (NA) of 0.85 is mounted inside the cryostat on the cold shield, having a temperature of about $30 \mathrm{~K}$.

To observe the SE, QDs are excited using a helium-neon (HeNe) laser with a wavelength of $633 \mathrm{~nm}$, and the $\mathrm{SE}$ is separated from the excitation by a colour filter Schott RG680, transmitting wavelengths above $680 \mathrm{~nm}$. We 
spectrally resolve the SE by imaging the sample near field (NF) onto the input slit (corresponding area indicated in Fig. 1c) of an imaging spectrometer, with a resolution of $11 \mu \mathrm{eV}$. Therefore, this geometry allows to image the SE along the $x$ direction and detect spectral images, as shown in Fig. 1d. The bottom and top stripes are due to the SE into the WG mode, coupled into free space by the bottom and top couplers, respectively. The central stripe is the free space emission at the excitation position, indicated by the red dot in Fig. 1c. Alternatively, omitting spectral resolution, the sample NF can be imaged in $x$ and $y$ onto a camera. Equivalently, for directional space imaging, the sample far field in the in-plane wavevector components $k_{x}$ and $k_{y}$ is imaged onto another camera.

\section{RESULTS}

\subsection{Loss}

We now discuss the determination of the loss coefficient $\alpha_{\mathrm{SI}}$ from spectral imaging. We introduce the distances $L_{\mathrm{t}}=L / 2-x$ and $L_{\mathrm{b}}=L / 2+x$ between the excitation position $x$ and the top and bottom couplers, respectively, as sketched in Fig. 2b, with the length $L$ of the WG, and t, b labelling the top and bottom couplers respectively. Using a Lambert-Beer extinction model, the SE powers emitted from the top and bottom couplers are proportional to $\eta_{i} \exp \left(-\alpha_{\mathrm{SI}} L_{i}\right)$ with $i \in\{\mathrm{t}, \mathrm{b}\}$, and the collection efficiency $\eta_{i}$ from the couplers. The present model assumes that most of the emission is locally excited, which we verified to be the case. ${ }^{15}$ To apply the model, we integrate the SE from the bottom and top couplers over the spectral range covered by the CCD camera of about $15 \mathrm{meV}$, to average over the possible directionality of the SE from individual QDs. Calling $P_{\mathrm{b}}^{\prime}$ and $P_{\mathrm{t}}^{\prime}$ the spectrally integrated emission, we calculate the ratio $P_{\mathrm{t}}^{\prime} / P_{\mathrm{b}}^{\prime}$ and plot it as a function of the excitation position $x$ in Fig. 2a. According to the above model, the ratio is given by

$$
\frac{P_{\mathrm{t}}^{\prime}}{P_{\mathrm{b}}^{\prime}}=\eta \exp \left(2 \alpha_{\mathrm{SI}} x\right)
$$

with $\eta=\eta_{\mathrm{t}} / \eta_{\mathrm{b}}$. The corresponding measurements, and fit according to Eq. (1), are shown in Fig. 2a for spectral ranges centered at four different energies. The random deviation of the data from the fit is possibly due to a residual influence of directional emission of the QDs. The resulting loss coefficient $\alpha_{\mathrm{SI}}$ is shown Fig. 2c, going from $0.02 \mu \mathrm{m}^{-1}$ (corresponding to $0.1 \mathrm{~dB} / \mu \mathrm{m}$ using $10 \mathrm{~dB}$ per decade intensity) at $E_{0}=1.378 \mathrm{eV}$, up to $\alpha_{\mathrm{SI}}=0.052 \mu \mathrm{m}^{-1}(0.2 \mathrm{~dB} / \mu \mathrm{m})$ at $E_{0}=1.425 \mathrm{eV}$. The extracted coefficient $\eta$ is shown in the inset of Fig. $2 \mathrm{c}$. We calculate an average value of $\eta=0.6 \pm 0.1$. We note that this factor also contains the coupling efficiencies into the spectrometer. Using direct imaging of the emission from the two couplers for excitation in the center of the WG, similar to the bright field reflection image shown in Fig. 1c, we find a factor $\eta=0.9 \pm 0.1$. The difference to the value determined from spectral imaging indicates that the latter is affected also by a somewhat unbalanced coupling efficiency into the spectrometer slit.

A second method to measure the WG loss we employed was to image the NF of the radiation inside the light cone along the WG, for a $100 \mathrm{fs}$ pulsed excitation onto the bottom coupler. Such pulses have a short coherence length, and interference effects from background scattering are reduced with respect to continuous wave excitation. In order to maximise the coupling to the fundamental mode, the excitation polarisation is set orthogonal to the propagation direction. To further reduce background scattering and remove the strong reflection and emission of the excitation from the couplers, we use a rectangular aperture centred on the WG. The measurements are shown in the top panel of Fig. 4 for different excitation energies $E_{0}$. The bottom coupler is located at $x=-13 \mu \mathrm{m}$, and light is propagating in the positive $x$ direction. The axis are calibrated using the distances between the couplers. For quantitative analysis, we calculate a NF profile as the difference of a line cut along the WG and one right next to the WG, as shown by dashed orange and white lines respectively. We fitted an exponential decay (red dashed line) in order to obtain the loss coefficient $\alpha_{\mathrm{NF}}$, which is shown as a function of energy by red circles in Fig. 2c. We observe an increase of $\alpha_{\mathrm{NF}}$ with the energy, which is attributed to radiation loss of the mode inside the light cone and to the absorption of the QD WL. Additionally, in the region of spectral overlap $\alpha_{\mathrm{NF}}$ is consistent with $\alpha_{\mathrm{SI}}$, which confirms our method. Finally, this also implies that the relative coupling efficiencies of the grating out-couplers can be determined by spectral imaging. 

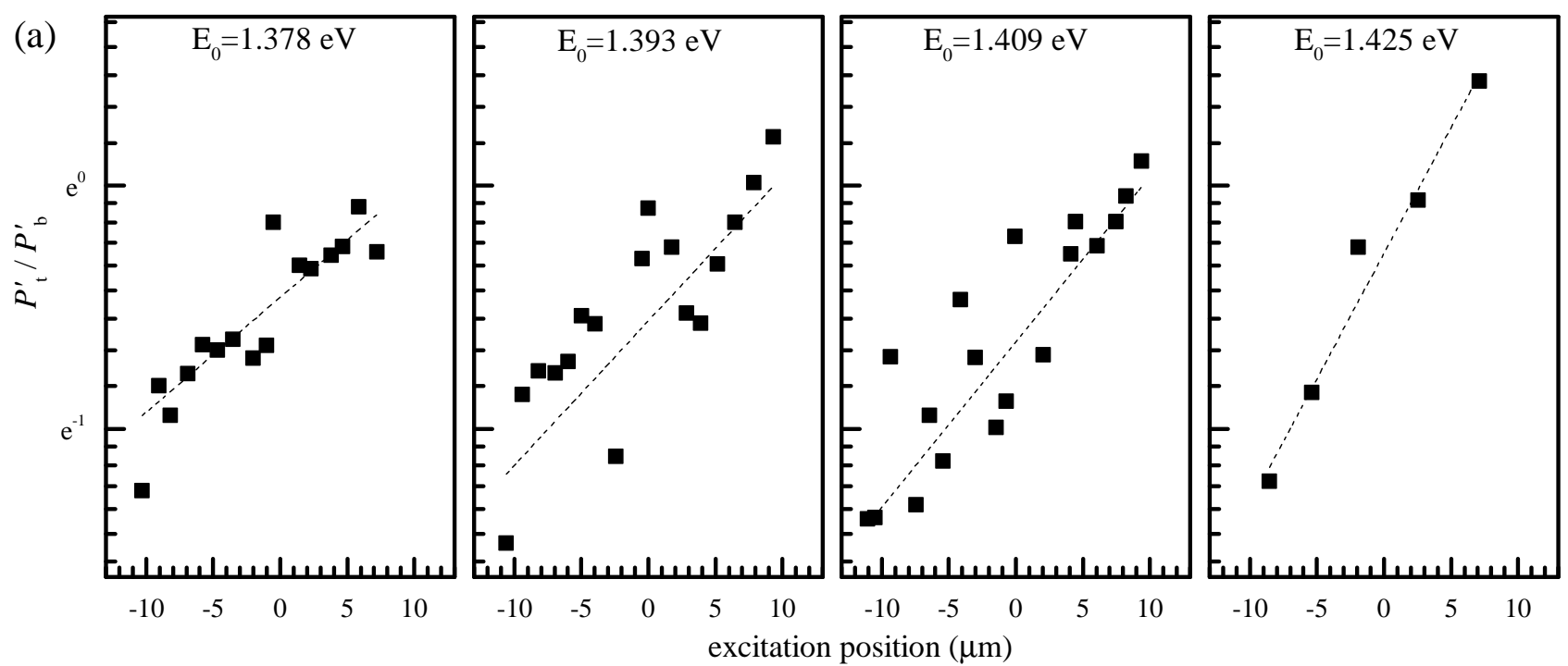

(b)

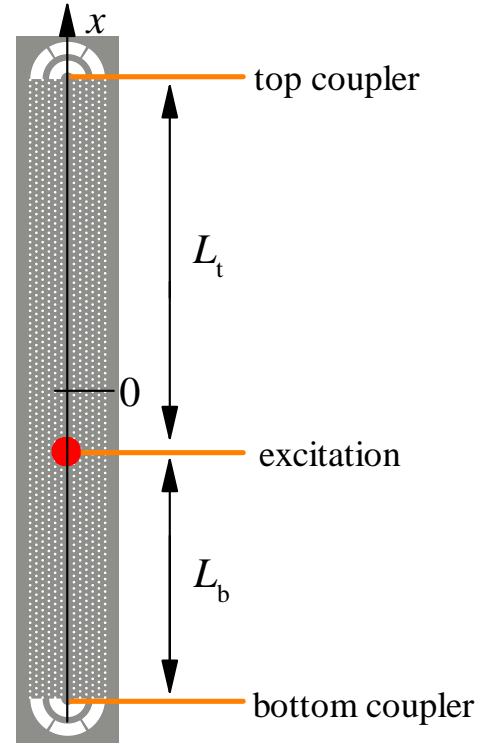

(c)

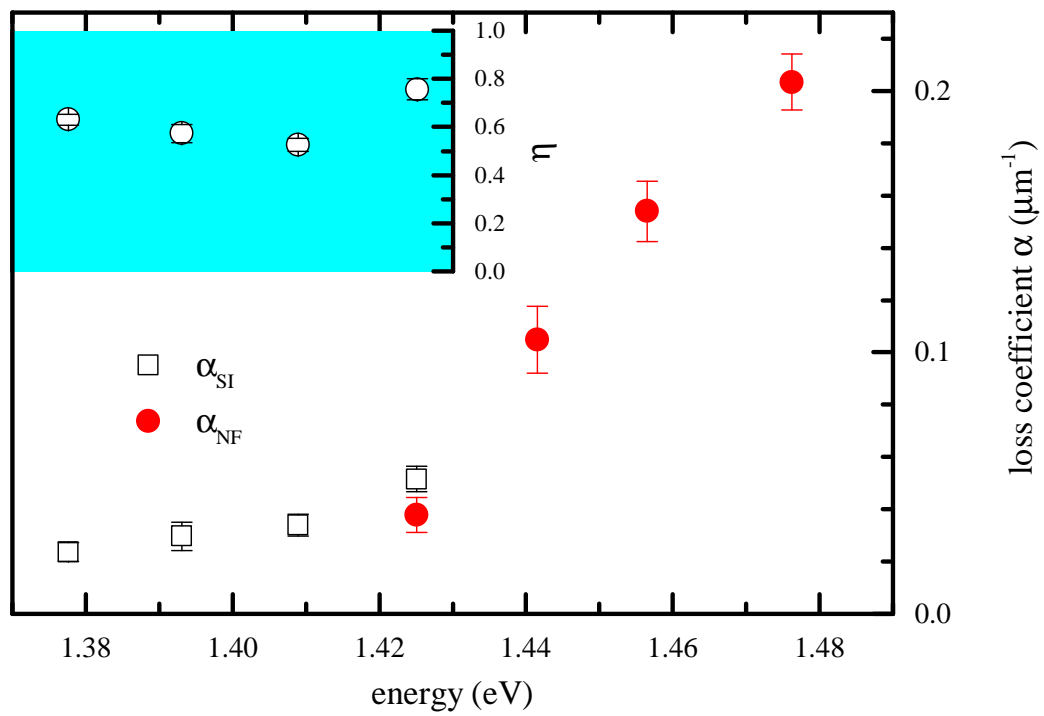

Figure 2. Determining the loss coefficient. (a) Ratio $P_{\mathrm{t}}^{\prime} / P_{\mathrm{b}}^{\prime}$ as a function of the excitation position along the waveguide for spectral ranges centred at different energies $E_{0}$, as indicated. Fits according to Eq. (1) are given as dashed lines. (b) Sketch of the geometry considered to estimate loss from SE measurements. (c) Propagation loss as a function of energy measured from SE and from NF; inset: relative collection efficiency from the couplers $\eta$ as a function of energy as obtained from SE spectral imaging measurements. 


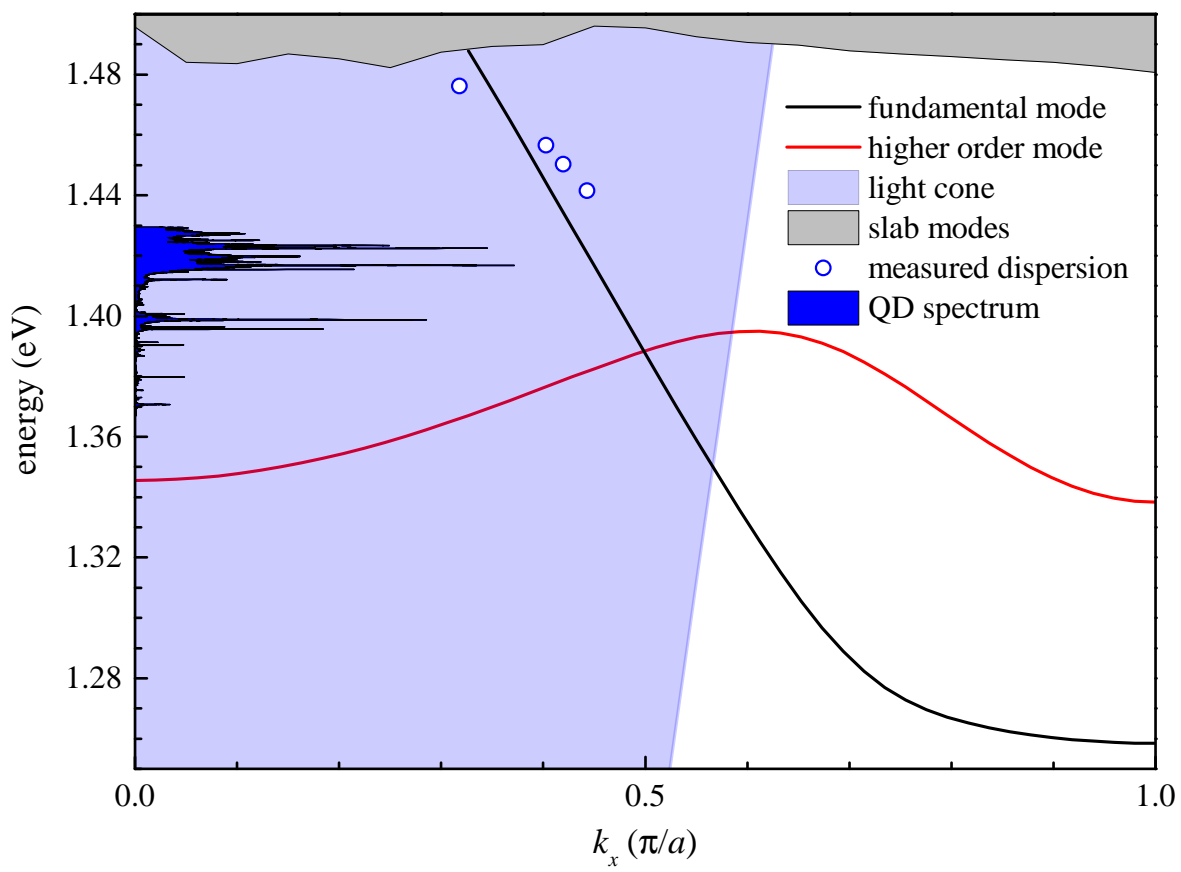

Figure 3. Simulated photonic band structure of the investigated WG, showing two guided modes, given in black and red lines, and the continuum above the band gap as gray area. Dark blue shaded area: example of QD SE spectrum from the bottom coupler, with excitation position at $-0.5 \mu \mathrm{m}$; blue circles: measured WG propagation wavevectors from $\mathrm{FF}$ imaging. All data for a temperature of $5 \mathrm{~K}$.

\subsection{Dispersion}

In Fig. 3, we show the simulated dispersion of the investigated WG, together with an emission spectrum from an ensemble of QDs. As discussed below, to match the measured dispersion, shown by the blue circles, an etching thickness parameter has been used in the simulation. The QDs emit between $1.37 \mathrm{eV}$ and $1.42 \mathrm{eV}$. The broader emission around $1.42 \mathrm{eV}$ is due to the WL. Sharp lines superimposed to the WL emission are attributed to localized excitons.

Experimentally determining the photonic band structure of the investigated sample is important for the understanding of experiments and for quantitative comparisons with numerical simulations. Different techniques have been applied, including near field scanning optical spectroscopy ${ }^{16}$ interferometric techniques, ${ }^{17}$ and transmission measurements.$^{18}$ Alternatively, the group index of the guided mode was measured using Fabry-Pérot fringes ${ }^{4}$ In this work, we measure the WG dispersion within the light cone by Fourier imaging ${ }^{19,20}$ which has been applied specifically to a WG system in Ref. 21.

From the FF images of the radiation loss along the WG, given in Fig. 4 bottom, the propagation wavevector of the corresponding WG mode within the light cone is determined. The measured FF profiles along the propagation direction correspond to the NF profiles shown in the top panel. The FF is calibrated using the visible $k$-space cut-off due to the acceptance angle of the MO, which is limited to $|\boldsymbol{k}| \leq k_{0} \mathrm{NA}$, with the free-space wavevector $k_{0}=2 \pi / \lambda$. The FF profile of the WG radiation loss is a stripe at definite values of $k_{x}$, elongated in $k_{y}$ direction. The position of the stripe along $k_{x}$ is the WG mode wavevector. The large extension in $k_{y}$ is reflecting the small extension of the mode in $y$. The finite width in $k_{x}$ instead is due to the decay of the NF profile along $x$, the finite aperture size along $x$, and the laser bandwidth, as discussed in detail in Ref. 15. There are other features visible in the FF images, namely in the center region, which is attributed to residual reflections of the excitation by the microscope objective lenses, and at large $k_{x}$, which is attributed to the remaining scattering from the excitation beam reflection at the sample, also visible in the real space images close to the bottom coupler. We extract the WG mode wavevector by taking a line cut along $k_{x}$ (as shown in the figure by the white dashed line), and by fitting the peak with a Lorentzian profile, as shown by the red dashed lines. The blue circles in Fig. 3 

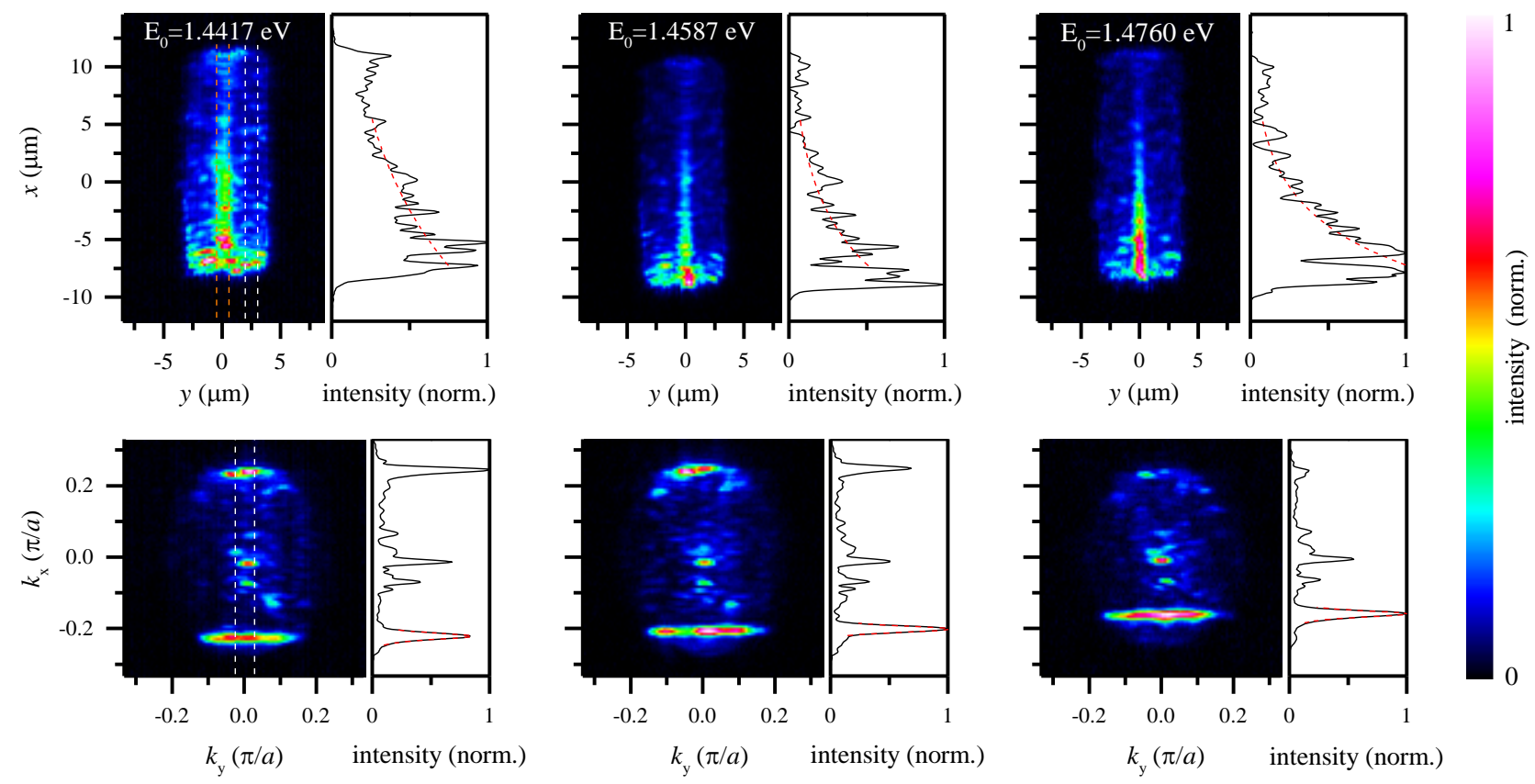

Figure 4. NF and FF imaging of the WG under pulsed excitation at the bottom coupler. Top: Normalized NF intensity of the out-of-plane loss of the WG propagating mode, at the energy indicated. Color scale as given. Profiles (black solid lines) are obtained as the difference between a region along the WG (orange dashed line) and a sideways displaced reference region (white dashed lines). Red dashed line: exponential fit. Bottom: corresponding FF imaging. Profiles (black solid lines) are obtained from a region highlighted by the white dashed line, and Lorentzian fits are given by the red dashed line.

are the resulting wavevectors as a function of the energy. Note that we show actually $-k_{x}$, using the reflection symmetry of the WG which results in a reflection symmetry of the dispersion around $k_{x}=0$. Interestingly, in the experiment, light is propagating with a positive group velocity $v_{\mathrm{g}}$ along the $k_{x}$ direction, while the measured propagation wavevector is negative. Although at first surprising, this is a simple consequence of the negative group velocity dispersion of the WG mode. In particular, in our experiment we impose a propagation direction such that $v_{\mathrm{g}}>0$, resulting in a negative propagation wavevector. Having measured the dispersion, we have used a single etching parameter $d$, which is removing a fixed thickness $d=8 \mathrm{~nm}$ from all surfaces of the structure, to match simulation and experiment. The refractive index of the structure, which is sometimes used in literature to adjust the simulations, has been taken from the well known data for GaAs at the measurement temperature of $5 \mathrm{~K}$. This is discussed in detail in Ref. 15.

\section{CONCLUSION}

In conclusion, we have determined the loss coefficient within the light cone by two different methods. Using spectral imaging of the quantum dot secondary emission, we find a loss coefficient, close to the light line, of about $0.02 \mu \mathrm{m}^{-1}\left(0.1 \mathrm{~dB} \mu \mathrm{m}^{-1}\right)$. Using near field and far field imaging of pulse propagation along the waveguide, we have determined the mode propagation wavevector as well as its loss, enabling to adjust the photonic simulations of the modes by matching a single thickness parameter. We emphasize that the spectral imaging using in the present experiment was used ${ }^{15}$ to measure beta factors up to $99 \%$, and the QD emission directionality, without assumptions on radiative rates into non-guided modes.

Information on the data underpinning the results presented here, including how to access them, can be found in the Cardiff University data catalogue at http://doi.org/10.17035/d.2019.0067928286. 


\section{REFERENCES}

[1] Lodahl, P., Mahmoodian, S., and Stobbe, S., "Interfacing single photons and single quantum dots with photonic nanostructures," Rev. Mod. Phys. 87, 347 (2015).

[2] Rigal, B., Dwir, B., Rudra, A., Kulkova, I., Lyasota, A., and Kapon, E., "Single photon extraction and propagation in photonic crystal waveguides incorporating site-controlled quantum dots," Appl. Phys. Lett. 112, 051105 (2018).

[3] Rao, V. S. C. M. and Hughes, S., "Single quantum-dot Purcell factor and $\beta$ factor in a photonic crystal waveguide," Phys. Rev. B 75, 205437 (2007).

[4] Arcari, M., Söllner, I., Javadi, A., Hansen, S. L., Mahmoodian, S., Liu, J., Thyrrestrup, H., Lee, E., Song, J., Stobbe, S., and Lodahl, P., "Near-unity coupling efficiency of a quantum emitter to a photonic crystal waveguide," Phys. Rev. Lett. 113, 093603 (2014).

[5] Lodahl, P., Mahmoodian, S., Stobbe, S., Rauschenbeutel, A., Schneeweiss, P., Volz, J., Pichler, H., and Zoller, P., "Chiral quantum optics," Nature 541, 473-480 (2017).

[6] Söllner, I., Mahmoodian, S., Hansen, S. L., Midolo, L., Javadi, A., Kiršanske, G., Pregnolato, T., El-Ella, H., Lee, E. H., Song, J. D., Stobbe, S., and Lodahl, P., "Deterministic photon-emitter coupling in chiral photonic circuits," Nat. Nanotech. 10, 775-778 (2015).

[7] Coles, R., Price, D., Dixon, J., Royall, B., Clarke, E., Kok, P., Skolnick, M., Fox, A., and Makhonin, M., "Chirality of nanophotonic waveguide with embedded quantum emitter for unidirectional spin transfer," Nat. Commun. 7, 11183 (2016).

[8] Javadi, A., Ding, D., Appel, M. H., Löbl, S. M. M. C., Söllner, I., Schott, R., Papon, C., Pregnolato, T., Stobbe, S., Midolo, L., Schröder, T., Wieck, A. D., Ludwig, A., Warburton, R. J., and Lodahl, P., "Spin-photon interface and spin-controlled photon switching in a nanobeam waveguide," Nat. Nanotech. 13, 398-403 (2018).

[9] Hughes, S., Ramunno, L., Young, J. F., and Sipe, J. E., "Extrinsic optical scattering loss in photonic crystal waveguides: Role of fabrication disorder and photon group velocity," Phys. Rev. Lett. 94, 033903 (2005).

[10] García, P. D., Javadi, A., Thyrrestrup, H., and Lodahl, P., "Quantifying the intrinsic amount of fabrication disorder in photonic-crystal waveguides from optical far-field intensity measurements," Appl. Phys. Lett. 102, 031101 (2013).

[11] Smith, C. J. M., Benisty, H., Olivier, S., Rattier, M., Weisbuch, C., Krauss, T. F., Rue, R. M. D. L., Houdré, R., and Oesterle, U., "Low-loss channel waveguides with two-dimensional photonic crystal boundaries," Appl. Phys. Lett. 77, 2813 (2000).

[12] Kuramochi, E., Notomi, M., Hughes, S., Shinya, A., Watanabe, T., and Ramunno, L., "Disorder-induced scattering loss of line-defect waveguides in photonic crystal slabs," Phys. Rev. B 72, 161318 (2005).

[13] Letartre, X., Seassal, C., Grillet, C., Rojo-Romeo, P., Viktorovitch, P., d'Yerville, M. L. V., Cassagne, D., and Jouanin, C., "Group velocity and propagation losses measurement in a single-line photonic crystal waveguide on InP membranes," Appl. Phys. Lett. 79, 2312 (2001).

[14] Rigal, F., Kristoffer, J. B., Lyasota, A., Jarlov, C., Dwir, B., Rudra, A., Kulkova, I., and Kapon, E., "Propagation losses in photonic crystal waveguides: Effects of band tail absorption and waveguide dispersion," Opt. Express 25, 28908 (2017).

[15] Scarpelli, L., Lang, B., Masia, F., Beggs, D., Muljarov, E., Young, A. B., Oulton, R., Höfling, S., Schneider, C., and Langbein, W., "99\% beta factor and directional coupling of quantum dots to fast light in photonic crystal waveguides determined by hyperspectral imaging," submitted (2019).

[16] Sukhorukov, A. A., Ha, S., Shadrivov, I. V., Powell, D. A., and Kivshar, Y. S., "Dispersion extraction with near-field measurements in periodic waveguides," Opt. Express 17, 3716 (2009).

[17] Gersen, H., Karle, T., Engelen, R., Bogaerts, W., Korterik, J., van Hulst, N., Krauss, T., and Kuipers, L., "Real-space observation of ultraslow light in photonic crystal waveguides," Phys. Rev. Lett. 94, 073903 (2005).

[18] Laucht, A., Günthner, T., Pütz, S., Saive, R., Frédérick, S., Hauke, N., Bichler, M., Amann, M.-C., Holleitner, A. W., Kaniber, M., and Finley, J. J., "Broadband Purcell enhanced emission dynamics of quantum dots in linear photonic crystal waveguides," J. Appl. Phys. 112, 093520 (2012). 
[19] Langbein, W., Hvam, J. M., and Zimmermann, R., "Time-resolved speckle analysis: A new approach to coherence and dephasing of optical excitations in solids," Phys. Rev. Lett. 82, 1040 (1999).

[20] Langbein, W. and Hvam, J. M., "Elastic scattering dynamics of cavity polaritons: Evidence for time-energy uncertainty and polariton localization," Phys. Rev. Lett. 88, 047401 (2002).

[21] Thomas, N. L., Houdré, R., Kotlyar, M. V., O’Brien, D., and Krauss, T. F., "Exploring light propagating in photonic crystals with Fourier optics," J. Opt. Soc. Am. B 24, 2964 (2007). 